\title{
Teratoma Mimicking Myelolipoma in Adrenal Region
}

\author{
Linika Agrawal ${ }^{1}$, Ahmed Khaled ${ }^{2}$, Tareak Al Nasir ${ }^{3}$, Waheed Zaman ${ }^{4}$
}

\begin{abstract}
A 19-year-old female presented with backache and left hypochondralgia on and off for one year. Complete blood counts, urinalysis and culture were negative. Ultrasonogram revealed normal kidney and urinary bladder along with a lobulated solid mass at left adrenal region with focal peripheral calcification. Based on the findings, CT scan was advised. CT scan without contrast showed a mass in left adrenal region of approx 3.3x3.2x2.6 cm with punctuate calcification in the wall as well as within the mass. The mass was well encapsulated with predominant fat attenuation interspersed with septae. The left adrenal was not separately identified, hence a presumptive diagnosis of adrenal myelolipoma was considered. The patient underwent left adrenalectomy and the specimen was sent for histopathological examination. Histopathology showed a mass adherent to normal adrenal showing several well differentiated ectodermal structures (tooth, hair follicles, sweat and sebaceous glands etc) consistent with a mature teratoma. The patient recovered uneventfully and is doing well at present.
\end{abstract}

\section{Keywords}

Teratoma, dermoid cyst, myelolipoma

\section{Introduction}

The vast majority of benign (mature) teratomas are cystic and are better known in clinical parlance as dermoid cysts. Primary benign retroperitoneal teratoma is a rare tumour in the adult population. In this case, we described a case of primary benign cystic teratoma located in the vicinity of the upper pole of left kidney (para adrenal) which was misdiagnosed as myelolipoma on imaging.

\section{Case Report:}

A 19-year-old female presented with pain in the left flank and back on and off since one year. The pain was dull aching in nature and used to aggravate on walking or jolting. There had been a few episodes of burning micturation as well. Complete blood counts, urinalysis and culture were negative. USG revealed a heterogenous retroperitoneal (a complex mass of anechoic and echogenic components) situated in between the aorta and upper pole of left kidney. The mass was lobulated, solid with focal peripheral calcification. Based on the findings, CT scan was advised. Ultra fast multidetector CT scan showed a well capsulated mass showing predominantly fat attenuation interspersed with septae. The mass revealed calcifications in the wall as well as within the lesion. As the left adrenal was not separately identified, a presumptive diagnosis of adrenal myelolipoma was considered. The patient underwent left adrenalectomy and the specimen was sent for histopathological examination(Fig:1). Histopathology showed several ectodermal structures (tooth, hair follicles, sweat and sebaceous glands, cartilage, tooth structures and stratified squamous epithelium etc), but no immature element, findings consistent with mature teratoma. The adrenal tissue was normal and adherent to the mass. The patient recovered uneventfully and is doing well at present. 


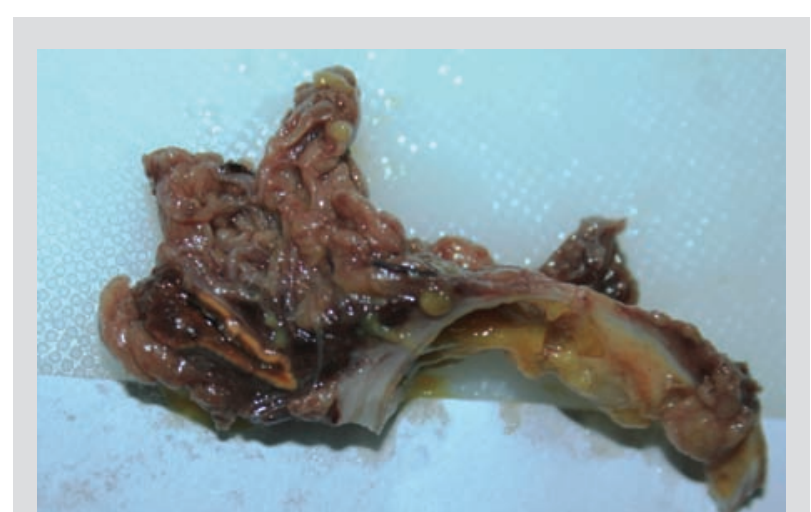

Fig 1: Adrenal gland with para-adrenal cystic mass

\section{Discussion:}

The great majority of all teratomas are composed of tissues representing at least two, but usually all three embryonic layers. Cystic teratomas usually contain only ectodermal element. If the neoplastic tissue is uniformly mature, the tumor is termed mature teratoma (almost always a dermoid cyst) but the presence of any immature tissue with an embryonal appearance warrants a designation of immature teratoma. Malignant transformation in a cystic teratoma may occur in foci of immature element and the usual type is a squamous cell carcinoma, though other type of malignancies can also be seen. Most of the teratomas are found in the gonads but approximately $3 \%$ of all teratomas occur at the extragonadal sites like retroperitoneum, floor of mouth, mediastinum, thyroid, urinary bladder and kidney. Retroperitoneal teratoma is rare and comprises of about $1 \%$ of all teratomas. ${ }^{1}$ Adult cases are rare, since most present in infancy and childhood.

Differentiation of a retroperitoneal teratoma in the para-adrenal location from a true adrenal teratoma is tricky. The clinical presentation ranges from symptomatic incidental mass to abdominal distention with or without pressure effect on adjacent organs. Para-adrenal teratomas usually present with hypochondralgia during exercise. ${ }^{2}$ A benign cystic teratoma in juxta-pancreatic location may also present as an inflammatory or neoplastic pancreatic cyst ${ }^{3}$ and in that location, Bacteriodes fragilis infection of the cyst is also reported. ${ }^{3}$

Para-adrenal teratoma poses a strong diagnostic challenge to radiology. Plain X-ray of abdomen alone can show mass with fat along with either calcification or bone. Likewise, ultrasonography shows uncomplicated fluid and calcification. These findings are better demonstrated by CT compared to ultrasound. Superior to CT scan is magnetic resonance imaging (MRI) which shows the characterstic signal of fat (hyperintensity in T1-weighted images) and water (hypointensity in T1-weighted images and hyperintensity in T-2 weighted images). In addition, MRI helps to distinguish between the tumor and adjacent structures through sagittal and coronal images.

Reports of teratoma in adrenal gland region are rare in the literature. ${ }^{4}$ Some of them have been diagnosed as lipomatous lesion of the adrenal gland on imaging. ${ }^{5}$ The lipomatous lesions of the adrenal gland include myelolipoma, lipoma, teratoma, angiomyolipoma and liposarcoma. ${ }^{6}$ These patients are mostly asymptomatic. Myelolipoma is the most common fatty tumor of the adrenal gland and was the presumptive diagnosis in our case. In contrast to myelolipoma, adrenal lipoma is very rare. The final diagnosis, however, still depends on the histopathological findings. We found features of teratoma on gross examination which was abutting the adrenal gland and was further confirmed on histopathology as mature cystic teratoma in para-adrenal region.

To our knowledge only few cases of teratoma in adrenal region have been reported. We believe that with the increasing use of modern imaging techniques, these unsuspected incidental tumors may become more prevalent than before. In particular, teratoma should be considered in the differential diagnosis of adrenal lipomatous tumor in all age groups. 


\section{References:}

1. Bhalla S, Misih K, Rana RS. Teratomas of rare sites: a review of ten cases. J Indian Med Assoc. 1991;89:291-4.

2. Hirano S,Kawaguchi S, Mikawa I . Primary retroperitoneal teratoma in an adult: ultrasonographic, computer tomographic and magnetic resonance imaging demonstrations. Hinyokika kiyo. 1998;34:2031-34.

3. Dewar G, Arnold M, Li AK. Retroperitoneal dermoid presenting as an infected pancreatic cyst. Aust NZ J Surg. 1990;60:488-9.
4. Lam KY, Lo CY. Teratoma in the region of adrenal gland: A unique entity masquerading as lipomatous adrenal tumor. Surgery. 1999;126:90-94.

5. Khong Pl, Lam KY, Ooi CG, Liu MJ, Metreweli C. Mature teratomas of the adrenal gland: imaging features. Abdom Imaging. 2002;27:347-350.

6. Kenney PJ, Wagner BJ, Rao P, Heffess CS. Myelolipoma: CT and pathologic features. Radiology. 1998;208:87-95. 